\title{
ON CARDINALITIES OF ULTRAPRODUCTS
}

BY H. JEROME KEISLER

Communicated by L. Henkin, February 27, 1964

Introduction. In the theory of models, the ultraproduct (or prime reduced product) construction has been a very useful method of forming models with given properties (see, for instance, [2]). It is natural to ask what the cardinality of an ultraproduct is when we are given the cardinalities of the factors. In this paper we obtain some new results in that direction; however, the questions stated explicitly in [2, p. 208], are still open.

Let us first mention briefly some of the known results. Throughout this note we shall let $D$ be a nonprincipal ultrafilter over a set $I$ of infinite power $\lambda$. Additional notation is explained in $\$ 1$ below.

1. $\alpha \leqq \alpha^{I} / D \leqq \alpha^{\lambda}[2$, p. 205].

2. If $D$ is not countably complete, then $\prod_{i \in I} \alpha_{i} / D$ is either finite or of power at least $2^{\omega}[2$, p. 208].

3. If $D$ is uniform, then $\lambda^{I} / D>\lambda$; moreover, $\left(2^{(\lambda)}\right)^{I} / D=2^{\lambda}$, where $2^{(\lambda)}=\sum_{\beta<\lambda} 2^{\beta}[2$, p. 206].

4. There exists a $D$ such that if $\alpha$ is infinite, then $\alpha^{I} / D=\alpha^{\lambda}[2, \mathrm{p}$. 207], [1, p. 399], and [3, p. 838]. (Two more general versions for products of cardinals are given in [1].)

We shall prove the following results.

Theorem A. (i) If $\alpha$ is infinite and $D$ is not countably complete, then

$$
\alpha^{I} / D=\left(\alpha^{I} / D\right)^{\omega} .
$$

(ii) For any $\alpha, \gamma$, and $D$,

$$
\left(\alpha^{\gamma}\right)^{I} / D \geqq\left(\alpha^{I} / D\right)^{\gamma} .
$$

(iii) If $D$ is uniform then

$$
\left(\alpha^{(\lambda)}\right)^{I} / D=\left(\alpha^{I} / D\right)^{\lambda}=\alpha^{\lambda}
$$

where $\alpha^{(\lambda)}=\sum_{\beta<\lambda} \alpha^{\beta}$.

We introduce the notion of a $(\beta, \gamma)$-regular ultrafilter in $\S 1$, and use it to prove Theorem $A$ and some more general results in $\$ 2$.

1. Regular ultrafilters. We shall adopt all of the set-theoretical notation introduced in [1], including the notions of an ultraproduct $\prod_{i \in I} \alpha_{i} / D$ and ultrapower $\alpha^{I} / D$ of the cardinals $\alpha_{i}, \alpha$. We denote the set of all functions on $X$ into $Y$ by ${ }^{x} Y$. We let $S(X)$ be the set of all 
subsets of $X$, and $S_{\beta}(\gamma)$ the set of all subsets of $\gamma$ of power less than $\beta$. Assume hereafter that $\beta, \gamma$ are infinite. If $f$ is a function on $X$ into $S(Y)$, then we define the function $f^{*}$ on $Y$ into $S(X)$ by

$$
f^{*}(y)=\{x \in X: y \in f(x)\}, \quad \text { for } y \in Y .
$$

Thus we always have $f^{* *}=f$.

Definition 1.1. Let $f$ be a function on $I$ into $S(\gamma)$. We shall say that $f$ makes $D(\beta, \gamma)$-regular if $f(i) \in S_{\beta}(\gamma)$ for all $i \in I$ and $f^{*}(\eta) \in D$ for all $\eta<\gamma . D$ is $(\beta, \gamma)$-regular if there exists an $f$ which makes $D(\beta, \gamma)$-regular.

Lemma 1.2. Let $g$ be a function on $\gamma$ into $S(I)$. Then $g^{*}$ makes $D(\beta, \gamma)$-regular if and only if $g(\eta) \in D$ for all $\eta<\gamma$ and $\bigcap_{\eta \in Y} g(\eta)=0$ for all $Y \subseteq \gamma$ of power $\beta$.

$D$ is said to be uniform if every member of $D$ is of power $\lambda$ (cf. [2]). As pointed out in [2], the uniform ultrafilters are the only interesting ones as far as the problems considered here are concerned.

Lemma 1.3. (i) If $D$ is not countably complete, then $D$ is $(\omega, \omega)$ regular.

(ii) If $\beta>\gamma$, then $D$ is $(\beta, \gamma)$-regular.

(iii) If $D$ is uniform, then $D$ is $(\operatorname{cf}(\lambda), \operatorname{cf}(\lambda))$-regular.

(iv) If $D$ is $(\operatorname{cf}(\gamma), \operatorname{cf}(\gamma))$-regular, then $D$ is $(\gamma, \gamma)$-regular.

REMARK. The notion of regularity has other simple properties which we shall not need here. For instance, if $D$ is $(\beta, \gamma)$-regular and $\beta \leqq \beta^{\prime}, \gamma \geqq \gamma^{\prime}$, then $D$ is $\left(\beta^{\prime}, \gamma^{\prime}\right)$-regular. If $\lambda, \beta<\gamma$, then $D$ is not $(\beta, \gamma)$ regular. Moreover, if $\lambda<\operatorname{cf}(\gamma)$, then $D$ is not $(\gamma, \gamma)$-regular.

The proofs of Lemmas 1.2 and 1.3 above may easily be supplied by the reader.

\section{Cardinality theorems.}

THEOREM 2.1. Suppose that $f$ makes $D(\beta, \gamma)$-regular, and let $\beta_{i}$ be the power of $f(i)$ for each $i \in I$. Then for any cardinals $\alpha_{i}, i \in I$, we have

$$
\prod_{i \in I}\left(\alpha_{i}^{\beta_{i}}\right) / D \geqq\left(\prod_{i \in I} \alpha_{i} / D\right)^{\gamma} .
$$

Proof. For each $i$, let $g_{i}$ be a one-one function on ${ }^{f(i)} \alpha_{i}$ into $\alpha_{i}^{\beta_{i}}$. Define the function $g$ on ${ }^{\gamma}\left(P_{i \in I} \alpha_{i}\right)$ into $P_{i \in I}{ }^{f(i)} \alpha_{i}$ by

$$
g\left(\left\langle h_{\eta}\right\rangle_{\eta<\gamma}\right)=k,
$$

where

$$
k(i)=g_{i}\left(\left\langle h_{\eta}(i)\right\rangle_{\eta \in \mathcal{S}(i)}\right) \quad \text { for each } i \in I .
$$


Now consider two arbitrary elements $h=\left\langle h_{\eta}\right\rangle_{\eta<\gamma}, \quad h^{\prime}=\left\langle h_{\eta}^{\prime}\right\rangle_{\eta<\gamma}$ of $\gamma\left(P_{i \in I} \alpha_{i}\right)$, and let $g(h)=k, g\left(h^{\prime}\right)=k^{\prime}$. Suppose that there exists $\eta<\gamma$ such that $h_{\eta} \neq{ }_{D} h_{\eta}^{\prime}$. Whenever $h_{\eta}(i) \neq h_{\eta}^{\prime}(i)$ and $\eta \in f(i)$ we have $k(i) \neq k^{\prime}(i)$. Since $f^{*}(\eta) \in D$ it follows that $k \neq_{D} k^{\prime}$. The desired inequality follows.

Theorem A follows from Lemma 1.3 and Theorem 2.1. Indeed we have the following more general result.

TheOREM 2.2. (i) If each cardinal $\alpha_{i}$ is infinite and $D$ is not countably complete, then

$$
\prod_{i \in I} \alpha_{i} / D=\left(\prod_{i \in I} \alpha_{i} / D\right)^{\omega}
$$

(ii) For any $\alpha_{i}, \gamma$, and $D$, we have

$$
\prod_{i \in I}\left(\alpha_{i}^{\gamma}\right) / D \geqq\left(\prod_{i \in I} \alpha_{i} / D\right)^{\gamma}
$$

(iii) If $D$ is uniform and $\operatorname{cf}(\gamma)=\operatorname{cf}(\lambda)$, then

$$
\prod_{i \in I}\left(\alpha_{i}^{(\gamma)}\right) / D \geqq\left(\prod_{i \in I} \alpha_{i} / D\right)^{\gamma} .
$$

(iv) If $D$ is uniform and, for each $i \in I,\left\{j \in I: \alpha_{j} \geqq \alpha_{i}\right\} \in D$, then

$$
\prod_{i \in I}\left(\alpha_{i}^{(\lambda)}\right) / D=\left(\prod_{i \in I} \alpha_{i} / D\right)^{\lambda}=\left(\prod_{i \in I} \alpha_{i}\right)^{\lambda} .
$$

PRoof. We observe that under the hypotheses of Theorem 2.1,

$$
\prod_{i \in I}\left(\alpha_{i}^{\left(\beta_{i}\right)}\right) / D \geqq\left(\prod_{i \in I} \alpha_{i} / D\right)^{\gamma} .
$$

Then (i), (ii), and (iii) follow using Lemma 1.3 with $\beta=\gamma=\omega, \beta=\gamma^{+}$, and $\beta=\gamma$, respectively. To prove (iv), we note that $\alpha_{i} \leqq \prod_{j \in I} \alpha_{j} / D$ for each $i \in I$, and hence

$$
\begin{aligned}
\left(\prod_{i \in I} \alpha_{i} / D\right)^{\lambda} & \leqq \prod_{i \in I}\left(\alpha_{i}^{(\lambda)}\right) / D \leqq \prod_{i \in I}\left(\alpha_{i}^{(\lambda)}\right) \leqq\left(\prod_{i \in I} \alpha_{i}\right)^{\lambda} \\
& \leqq\left(\prod_{i \in I}\left(\prod_{j \in I} \alpha_{j} / D\right)\right)^{\lambda}=\left(\prod_{i \in I} \alpha_{i} / D\right)^{\lambda}
\end{aligned}
$$

Theorem 2.3. Suppose that each $n_{i}$ is a finite cardinal and $D$ is not 
countably complete. Let $q \in{ }^{\omega} \omega$ be such that $\lim _{m \rightarrow \infty} q(m)=\infty$. If $\prod_{i \in I} n_{i} / D$ is infinite, then

$$
\prod_{i \in I}\left(n_{i}^{q\left(n_{i}\right)}\right) / D \geqq\left(\prod_{i \in I} n_{i} / D\right)^{\omega}
$$

Proof. Since $\prod_{i \in I} n_{i} / D$ is infinite, we have $\left\{i \in I: n_{i}>m\right\} \in D$ for each $m<\omega$. Let $f$ be the function on $I$ into $S_{\omega}(\omega)$ defined by

$$
f(i)=\left\{0,1, \cdots, q\left(n_{i}\right)-1\right\}, \quad \text { for each } i \in I .
$$

For each $r<\omega$, there is a greatest $m<\omega$ such that $q(m) \leqq r$, and hence

$$
f^{*}(r)=\left\{i \in I: q\left(n_{i}\right)>r\right\} \supseteq\left\{i \in I: n_{i}>m\right\} \in D .
$$

Thus $f$ makes $D(\omega, \omega)$-regular. The result now follows from Theorem 2.1 with $\beta=\gamma=\omega$.

Notice that the result 2 stated in the introduction follows from the above theorem, because if $\prod_{i \in I} \alpha_{i} / D$ is infinite then we may choose $n_{i}$ such that $n_{i}^{n_{i}} \leqq \alpha_{i}$ and $\prod_{i \in I} n_{i} / D$ is infinite.

We conclude with some historical remarks. The $(\omega, \lambda)$-regular ultrafilters have been considered in the literature, for instance in $[1],[2]$, [3], [6]. The result 4 stated in the introduction was shown in [1], $[2],[3]$ to hold for all $(\omega, \lambda)$-regular ultrafilters $D$. It is not difficult to show that any ultrafilter $D$ which belongs to the class $Q\left(\alpha^{+}\right)$defined in [4] is $(\omega, \alpha)$-regular. However, by Theorem 5.1 of [5], there is an $(\omega, \lambda)$-regular $D$ which is not a member of $Q\left(\omega_{2}\right)$.

\section{REFERENCES}

1. C. C. Chang and H. J. Keisler, Applications of ultraproducts of pairs of cardinals to the theory of models, Pacific J. Math. 12 (1962), 835-845.

2. T. Frayne, A. C. Morel, and D. Scott, Reduced direct products, Fund. Math. 51 (1962), 195-228.

3. H. J. Keisler, Limit ultrapowers, Trans. Amer. Math. Soc. 107 (1963), 382-408.

4. - Ultraproducts and elementary classes, Indag. Math. 23 (1961), 477-495.

5. - Good ideals in fields of sets, Ann. of Math. (2) 79 (1964), 338-359.

6. S. B. Kochen, Ultraproducts in the theory of models, Ann. of Math. (2) 74 (1961), 221-261.

UNIVERSITY OF WisCONSIN 